Discussion Even though the focus group procedures varied, there was significant overlap and repetition in the feedback received on the same guideline resources. The patient focus group facilitated by a clinician engaged participants in discussions oriented to clinical issues. The comic book was considered to be a novel communication vehicle by clinicians but not so by public.

Implications for Guideline Developers/Users Involving a motivated Lay Committee facilitated by someone not directly related to the project seems to be a valuable alternative to other focus groups of patients which may require more effort and resources.

\section{IMPLEMENTING NUTRITION GUIDELINES THROUGH PRACTICE TOOLS FOR REGISTERED DIETITIANS}

K Kren, L Moloney, P Ziegler, A Acosta. Academy of Nutrition and Dietetics, Chicago, USA

\section{0:1136/bmjqs-2013-002293.84}

Background Evidence Based Dietetics Practice Toolkits are resources intended to assist registered dietitians (RDs) in implementing nutrition guidelines derived from systematic reviews.

Objectives Surveys, sent at least one year past publication, were used to explore who uses toolkits, how toolkits are used, and if RDs find toolkits useful for implementation of guidelines.

Methods A standard questionnaire was sent to 1379 individuals for six toolkits (Celiac Disease, Critical Illness, Diabetes, Heart Failure, Oncology, and Paediatric Overweight) in 2011 and 2012, using Survey Monkey.

Results Responses received were 131 (9\%), of which 42\% were RDs in practice $>15$ years, in direct care (63\%), and in settings of outpatient (51\%) and inpatient (31\%). Respondents mostly used toolkits for patient care (66\%), nutrition counselling (40\%) and development of forms (37\%). Regarding the tools provided, $66 \%$ of respondents indicated that they found the summary of nutrition care and patient education materials to be very/somewhat useful or useful. The same was true for $63 \%$ for the case studies, $61 \%$ for the flowchart of patient encounters, $60 \%$ for the patient encounter process instructions and $56 \%$ for the sample documentation forms. Some respondents (24\%) seldom or never used the guidelines prior to toolkit use; however, 65\% indicated that the toolkit was useful in translating the guidelines to practice.

Discussion The implementation of guidelines is often overlooked yet is crucial to changing practice. These toolkits allow a useful strategy for assisting with implementation. Similar practice tools, tailored to practitioners and setting, may be useful in guideline implementation for a variety of conditions.

\section{TRANSFORMING EVIDENCE FROM MULTIPLE GUIDELINES INTO USER FRIENDLY CLINICAL PRACTICE TOOLS FOR REMOTE CANCER TREATMENT-RELATED SYMPTOM MANAGEMENT: THE COSTARS PROJECT}

${ }^{1,2} \mathrm{D}$ Stacey, ${ }^{2,3} \mathrm{M}$ Carley, ${ }^{3} \mathrm{M}$ Harrison, ${ }^{4}$ Pan-Canadian Oncology Symptom Triage and Remote Support (COSTaRS), Group. 'University of Ottawa, Ottawa, Canada; ${ }^{2}$ Ottawa Hospital Research Institute, Ottawa, Canada; ${ }^{3}$ Queen's University, Kingston, Canada; ${ }^{4}$ Canada

\section{0:1136/bmjqs-2013-002293.85}

Objectives To transform evidence from guidelines for clinical practice tools for remote management of patients experiencing symptoms related to cancer treatment. Clinical practice protocols are defined as user-friendly knowledge translation tools to support patient care. These tools narrow the know-do gap by presenting the best available evidence from guidelines while using a format that is sensitive to how nurses think and what nurses do. Methods Mixed methods descriptive study guided by CANIMPLEMENT $\odot$. The process involved: a) conducting a systematic search for guidelines; b) developing symptom-specific protocols using evidence from quality appraised clinical practice guidelines; c) reaching consensus on the clinical practice protocol template, and d) validating the clinical practice protocols.

Results Clinical practice protocols were developed and validated for 13 symptoms using 42 clinical practice guidelines with a median of 3 guidelines per protocol (range 1 for bleeding to 7 vomiting). For the first two protocols, source guideline AGREE rigour subscale ratings ranged from $8 \%$ to $86 \%$ (median 60.1; diarrhoea; 40.5 fever). The protocols were developed using guidelines, symptom severity questions included the Edmonton Symptom Assessment System, and iterative feedback from practicing nurses. Usability testing revealed: high readability, just the right amount of information, and appropriate terms. Access to protocols needs to be tailored to individual practices (e.g. electronic application, access to paper-based versions). Nurses requested training and support to implement them.

Discussion These tools, created from guidelines, transform evidence into user-friendly protocols for use by nurses when guiding patients at home to better manage their cancer treatmentrelated symptoms.

\section{USE OF NON-RANDOMISED STUDIES IN THE GUIDELINE PROCESS: THE GRADE APPROACH}

N Santesso, R Mustafa, H Schunemann, C4-GEP Methodology Group. McMaster University, Hamilton, Canada

\section{0:1136/bmjqs-2013-002293.86}

Background The GRADE approach to guideline development requires a review of the best available evidence which includes randomised controlled trials (RCTs) and non-randomised studies (NRS).

Objectives Describe the use of NRS as a replacement, a sequence, or a complement for RCTs, in a World Health Organization guideline using the GRADE approach.

Methods We searched the literature using no study type limits for the effect of screening and treatment of precancerous lesions on patient or population important outcomes and for baseline risks. We assessed quality of the evidence using GRADE.

Results Depending on the outcomes, we found few to no RCTs. When there was low/very low overall quality evidence from RCTs, we used NRS studies with no independent control groups to compare proportions between groups and calculate a relative effect of treatment and this evidence replaced the RCT evidence with similar/higher quality evidence. We found no evidence in RCTs for long-term outcomes, such as spontaneous abortion. Therefore, we used data from NRS (cohort studies) for premature delivery (a surrogate) to provide sequential evidence. For evidence about baseline risk of precancerous lesions and other outcomes, we used NRS a complement to the RCT data.

Discussion Data from NRS provided evidence in three ways. One key criterion to consider when grading this evidence is indirectness due to indirect comparisons, surrogate outcomes or varying population risks. 
Implications for Guideline Developers/Users Guideline developers can use GRADE and these methods when there is no evidence or low/very low quality evidence from RCTs.

\section{ADDRESSING CONTINUOUS DATA FOR PARTICIPANTS EXCLUDED FROM TRIAL ANALYSIS: A GUIDE}

${ }^{1} S$ Ebrahim, ${ }^{2} E$ Akl, ${ }^{5} \mathrm{R}$ Mustafa, ${ }^{3} \mathrm{X}$ Sun, ${ }^{1} \mathrm{~S}$ Walter, ${ }^{1} \mathrm{D}$ Heels-Ansdell, ${ }^{4} \mathrm{P}$ Alonso-Coello, ${ }^{1} \mathrm{~B}$ Johnston, ${ }^{1} \mathrm{G}$ Guyatt. ${ }^{1}$ McMaster University, Hamilton, Canada; ${ }^{2}$ American University of Beirut, Beirut, Lebanon; ${ }^{3}$ The Centre for Health Research, Kaiser Permanente Northwest, Portland, USA; ${ }^{4}$ beroamerican Cochrane Centre, CIBERESP-IIB Sant Pau, Barcelona, Spain; ${ }^{5}$ University of Missouri, Kansas City, USA

\section{0:1136/bmjgs-2013-002293.87}

Background Guideline developers addressing quality of evidence commonly confront studies with missing data.

Objectives To develop a framework for assessing risk of bias resulting from missing participant data for continuous outcomes in systematic reviews.

Methods We developed a range of progressively more stringent imputation strategies to challenge the robustness of the pooled estimates. We applied our approach to two systematic reviews.

Results We used 5 sources of data for imputing means for participants with missing data: [A] the best mean score among the intervention arms of included trials, [B] the best mean score among the control arms of included trials, [C] the mean score from the control arm of the same trial, [D] the worst mean score among the intervention arms of included trials, [E] the worst mean score among the control arms of included trials. Using these sources of data, we developed four progressively more stringent imputation strategies. In the first example review, effect estimates were diminished and lost significance as the strategies became more stringent, suggesting the need to rate down confidence in estimates of effect for risk of bias. In the second review, effect estimates maintained statistical significance using even the most stringent strategy, suggesting missing data does not undermine confidence in the results.

Discussion Our approach provides rigorous yet reasonable and relatively simple, quantitative guidance that guideline developers can use for judging the impact of risk of bias as a result of missing participant data in systematic reviews of continuous outcomes.

\section{HANDLING TRIAL PARTICIPANTS WITH MISSING DATA IN META-ANALYSES OF DICHOTOMOUS OUTCOMES: GUIDANCE FOR SYSTEMATIC REVIEWERS}

${ }^{1} \mathrm{E}$ Akl, ${ }^{2,4} \mathrm{~B}$ Johnston, ${ }^{3} \mathrm{P}$ Alonso-Coello, ${ }^{4} \mid$ Neumann, ${ }^{4} \mathrm{~S}$ Ebrahim, ${ }^{5} \mathrm{M}$ Briel, ${ }^{4} \mathrm{D}$ Cook, ${ }^{4} \mathrm{G}$ Guyatt. ${ }^{1}$ American University of Beirut, Beirut, Lebanon; ${ }^{2}$ Sick Kids Research Institute, Toronto, Canada; ${ }^{3}$ Ibero-American Cochrane Centre, Barcelona, Spain; ${ }^{4}$ McMaster University, Hamilton, Ontario; ${ }^{5}$ Basel Institute for Clinical Epidemiology, Basel, Switzerland

\section{0:1136/bmjqs-2013-002293.88}

Background Systematic reviewers including all randomised participants in their meta-analyses need to make assumptions about the outcomes of those with missing data.

Objectives To provide systematic review authors with guidance on dealing with participants with missing data for dichotomous outcomes.
Methods The authors used an iterative process of suggesting guidance and obtaining feedback to arrive at a proposed approach.

Results For participants with missing data, systematic reviewers can use a range of plausible assumptions in the intervention and control arms. Extreme assumptions include 'all' or 'none' of the participants had an event, but these assumptions are not plausible. Less extreme assumptions may draw on the incidence rates within the trial (e.g., same incidence in the trial control arm) or in all trials included in the meta-analysis (e.g., highest incidence among control arms of all included trials). The primary metaanalysis may use either a complete case analysis or a plausible assumption. Sensitivity meta-analyses to test the robustness of the primary meta-analysis results should include extreme plausible assumptions. When the meta-analysis results are robust to extreme plausible assumptions, inferences are strengthened. Vulnerability to extreme plausible assumptions suggests rating down confidence in estimates of effect for risk of bias.

Conclusions This guide proposes an approach to establishing confidence in estimates of effect when systematic reviewers are faced with missing participant data for binary dichotomous outcomes in randomised trials.

\section{ASSESSMENT OF THE EVIDENCE FOR DIAGNOSTIC TESTS AND STRATEGIES: A SYSTEMATIC REVIEW OF AVAILABLE TOOLS}

${ }^{1,2} \mathrm{R}$ Mustafa, ${ }^{1,6,7} \mathrm{~W}$ Wiercioch, ${ }^{1} \mathrm{M}$ Falavigna, ${ }^{1} \mathrm{Y}$ Zhang, ${ }^{3} \mathrm{~B}$ Prediger, ${ }^{4} \mathrm{~A}$ Cheung, ${ }^{1} \mathrm{~L}$ Ivanova, ${ }^{5}$ I Arevalo-Rodriguez, ${ }^{1} \mathrm{H}$ Schünemann, on behalf of the DU-Diagnosis SR group. ${ }^{1}$ McMaster University, Hamilton, Canada; ${ }^{2}$ University of Missouri-Kansas City, Kansas City, USA; ${ }^{3}$ Germany; ${ }^{4}$ University of British Columbia, Vancouver, Canada; ${ }^{5}$ Universidad Nacional de Colombia, Bogota, Columbia; ${ }^{6}$ Universidade Federal do Rio Grande do Sul, Porto Alegre, Brazil; ${ }^{7}$ National Institute of Science and Technology for Health Technology Assessment, Porto Alegre, Brazil

\section{0:1136/bmjqs-2013-002293.89}

Background The challenges facing guideline developers when making recommendations about diagnostic tests and strategies (DTS) are considerably different when compared to treatment recommendations.

Objectives To identify, describe and compare all available instruments, checklists, critical appraisal tools, and indices designed for assessing the quality of evidence (QoE) or strength of recommendations (SoR) dealing with diagnostic tests and strategies.

Methods We conducted a comprehensive systematic search of the literature including state of the art diagnostic guidelines, methods papers and diagnostic systematic reviews.

Results We identified 45 tools and modifications of existing tools to assess the QoE and SoR of DTS. Most tools acknowledge the importance of assessing the QoE and SoR separately. Most tools include individual quality criteria and study design but no tool rates all quality criteria suggested by the GRADE working group. Only two tools explicitly consider factors that increase the confidence in the evidence. When moving from evidence to recommendations, patient values and preferences and resources were rarely considered.

Discussion There is confusion about the terminology that describes the various factors that influence the QoE and SoR. The criteria for evaluating the QoE and moving from evidence to recommendations are incomplete for most guideline development frameworks that we evaluated.

Implications for Guideline Developers/Users The GRADE approach is the most complete approach encompassing all factors but users will benefit from a better description of the 